\title{
Analysis of Variability in HIV-1 Subtype A Strains in Russia Suggests a Combination of Deep Sequencing and Multitarget RNA Interference for Silencing of the Virus
}

\author{
Olga V. Kretova, ${ }^{\star}$ Vladimir R. Chechetkin, ${ }^{*}$ Daria M. Fedoseeva, \\ Yuri V. Kravatsky, Dmitri V. Sosin, Ildar R. Alembekov, Maria A. Gorbacheva, \\ Natalya M. Gashnikova, and Nickolai A. Tchurikov
}

\begin{abstract}
Any method for silencing the activity of the HIV-1 retrovirus should tackle the extremely high variability of HIV-1 sequences and mutational escape. We studied sequence variability in the vicinity of selected RNA interference (RNAi) targets from isolates of HIV-1 subtype A in Russia, and we propose that using artificial RNAi is a potential alternative to traditional antiretroviral therapy. We prove that using multiple RNAi targets overcomes the variability in HIV-1 isolates. The optimal number of targets critically depends on the conservation of the target sequences. The total number of targets that are conserved with a probability of $0.7-0.8$ should exceed at least 2. Combining deep sequencing and multitarget RNAi may provide an efficient approach to cure HIV/AIDS.]
\end{abstract}

Keywords: HIV-1 in Russia, deep sequencing, RNAi, multiple targeting

\section{Introduction}

$\mathbf{R}$ NA INTERFERENCE (RNAI)-based technology is a potential alternative to traditional antiretroviral therapy. The most prevalent and ancient viral infections in humansfor example, influenza, hepatitis $\mathrm{C}$ virus, and more recently, HIV/AIDS - escape current antiviral drugs due to the high mutation rate of the virus. Development of a suitable antiviral RNAi approach should take into account the variability of the viral genome in the selected targets of the viral transcripts. Short interfering RNAs (siRNAs) that are perfectly complementary to their corresponding targets, have been shown to function as potent inhibitors of virus replication. ${ }^{1-3}$ Therefore, monitoring of nucleotide sequences of the selected targets in the viral RNA should be performed to properly design siRNAs. Next-generation sequencing techniques could potentially be used to study the variability in the current isolates of a particular virus for a particular population, or even of an infected individual. In the latter case, the detected variability of the viral genome can also be very high. ${ }^{4,5}$ In this study, we describe the variability of the current HIV-1 subtype $\mathrm{A}$ in five isolates in Russia, and we also suggest the use of several RNAi targets for the development of efficient RNAi technology for the treatment of HIV/AIDS. Our data indicate that efficient RNAi for silencing of HIV-1 needs at least two targets. We believe that this strategy is not only limited to HIV-1 but could also be used for the development of similar technologies against other highly mutated viruses.

\section{Materials and Methods}

\section{Collection of samples and virus isolation}

RNA preparations were provided by the State Research Center of Virology and Biotechnology Vector (Russia) from their collection of isolates. Five isolates of HIV-1 subtype A (10RU6587, 11RU6933, 11RU6949, 10RU6483, and 11RU1996), which were collected by N.M. Gashnikova, were used. RNA was extracted from $500 \mu \mathrm{L}$ of plasma samples using ViroSeq reagents (Celera Diagnostics), and then treated with DNase using a Turbo DNA-free kit (Ambion). The concentration of RNA preparations was measured using a NanoDrop 2000.

\section{Reverse transcription polymerase chain reaction $(R T-P C R)$}

Five RNA preparations from five isolates were pooled (6 ng of each) and used for RT-PCR. Approximately $15 \mathrm{ng}$ of total RNA and M-MLV reverse transcriptase were used to synthesize cDNAs by the use of a Turbo DNA-free kit (Ambion) according 
Table 1. Primers Used for Reverse Transcription Polymerase Chain Reaction

\begin{tabular}{|c|c|c|c|}
\hline No & Text of primer $\left(5^{\prime}-3^{\prime}\right)$ & Used for & Domain \\
\hline 1 & TTTGATATGTCCATTGGTCTAGCCCTTGTT & Primer extension, $(-)$ primer & RT-A1 \\
\hline 2 & ACAAAGATCTTAGAGCCCTTTAGAG & PCR-1, (+) primer & RT-A1 \\
\hline 3 & TACTTCTGCTATTAАTTСТTTTGATGG & PCR-1, (-) primer & RT-A1 \\
\hline 4 & CATCTATTGAGATGGGGATTTACCA & PCR-2, $(+)$ primer & RT-A1 \\
\hline 5 & TGTTCTCTGCCAATTCCAATTCTG & PCR-2, (-) primer & RT-A1 \\
\hline 6 & CTCTGTTAGTGCTTTGGTCCCCCTAAGGAG & Primer extension, $(-)$ primer & $\mathrm{RT}-\mathrm{A} 2$ \\
\hline 7 & GAAAGTATACTGCATTCACTATACC & PCR-1, (+) primer & $\mathrm{RT}-\mathrm{A} 2$ \\
\hline 8 & TGACTTGCCCAATTTAGTTTTCCC & PCR-1, (-) primer & RT-A2 \\
\hline 9 & GACAAAGATCTTAGAGCCCTTTAGA & PCR-2, (+) primer & RT-A2 \\
\hline 10 & TATATCATTGACAGTCCAGCTTTCC & PCR-2, (-) primer & $\mathrm{RT}-\mathrm{A} 2$ \\
\hline 11 & TTCTTGGTACTACCTTTATTTCGTTATTGTCTTG & Primer extension, $(-)$ primer & Int-A3 \\
\hline 12 & GGCCAGTAAAAGTAATACATACAGA & PCR-1, (+) primer & Int-A3 \\
\hline 13 & CTTCACCTTTCCAGAGTAGCTTTG & PCR-1, (-) primer & Int-A 3 \\
\hline 14 & AGAATTTGGAATTCCCTACAATCCC & PCR-2, $(+)$ primer & Int-A3 \\
\hline 15 & TCTGCTGTCCCTGTAATAAACCC & PCR-2, (-) primer & Int-A3 \\
\hline 16 & CTAGAATCATTTCTTGTGGGTTGGGGTCTGTG & Primer extension, $(-)$ primer & Vpu-A4 \\
\hline 17 & GGATCATCAAAATCCTATACCAAAG & PCR-1, (+) primer & Vpu-A4 \\
\hline 18 & TACACAGGCATGTGTAGCCCAG & PCR-1, (-) primer & Vpu-A4 \\
\hline 19 & AGTAGGACTAATAGTAGCATTCATAG & PCR-2, (+) primer & Vpu-A4 \\
\hline 20 & TAATAGACTGTGACCCACAAGTTATTT & PCR-2, (-) primer & Vpu-A4 \\
\hline 21 & TCCTAGGTGATATGGCCTGGTGTACCATTTG & Primer extension, $(-)$ primer & Gag-A6 \\
\hline 22 & AAAGTTACAGAGGAGATCTCTCGA & PCR-1, (+) primer & Gag-A6 \\
\hline 23 & CCTGGGTGCCTTTCACCTCTAT & PCR-1, (-) primer & Gag-A6 \\
\hline 24 & TTGACTAGCGGAGGCTAGAAGG & PCR-2, (+) primer & Gag-A6 \\
\hline 25 & ATTATGTAATGATTTAAGTTCTTCTGTT & PCR-2, (-) primer & Gag-A6 \\
\hline
\end{tabular}

Sequences 1-20 or 21-25 were selected using the corresponding regions in AF316544 or AF286250, respectively.

to the manufacturer's instructions. cDNAs corresponding to five regions in the viral RNA were synthesized using the primers indicated in Table 1. Nested PCR was used for amplification of regions of $\sim 300 \mathrm{bp}$ that contained the selected RNAi targets. ${ }^{6}$ Primers were selected using the Primer Selection Tool (http:// biotools.umassmed.edu/). The conditions for PCR for each set of primers were determined in preliminary experiments using a Mastercycler personal PCR instrument (Eppendorf). The identity of amplified DNA fragments was confirmed by cloning and sequencing. RT-PCR products were cloned into the pGEMT Easy Vector (Promega) and sequenced. In total, 42 sequences of $\sim 300$ bp were deposited in GenBank Nucleotide Sequence Database with Accession Numbers KC681847-KC681888. The study was approved by the Institutional Review Board of the Engelhardt Institute of Molecular Biology of Russian Academy of Sciences.

\section{Luciferase reporter assays}

HEK293T cells were plated 1 day before transfection $\left(5 \times 10^{5}\right.$ cells per $60-\mathrm{mm}$ culture dish). To prepare liposomes, $10 \mathrm{ng}$ of the experimental DNA constructs containing the targets of RNAi cloned into the psiCHECK-2 (Promega) vector were mixed with $100 \mathrm{ng}$ of the corresponding DNA constructs that expressed the RNA hairpins cloned into the GeneClipU1 Neomycin vector (Promega) in $600 \mu \mathrm{L}$ of serum-free medium. Next, $1 \mu \mathrm{L}$ of TransFast reagent (Promega), diluted according to the manufacturer's recommendations, was added, and the mixture was incubated at room temperature for $15 \mathrm{~min}$. The cell suspension $(0.2 \mathrm{~mL}$, $\sim 5 \times 10^{4}$ cells) was centrifuged at $2,000 \mathrm{rpm}$ in $1.5-\mathrm{mL} \mathrm{Ep}-$ pendorf tubes for $3 \mathrm{~min}$ at room temperature, and the pre- cipitate was mixed with $60 \mu \mathrm{L}$ of the liposome-containing sample. After incubation for $1 \mathrm{~h}$ at $37^{\circ} \mathrm{C}$, the transfected cells were transferred into a 24 -well plate containing $0.5 \mathrm{~mL}$ per well of the cell culture medium supplemented with serum, and cells were incubated for $72 \mathrm{~h}$. Firefly and Renilla luciferase activities were measured using the Dual-Luciferase Reporter Assay System (Promega) and a Reporter Microplate Luminometer (Turner BioSystems). The Renilla luciferase data were normalized to the firefly luciferase data. Excel and Origin software were used for data analysis.

\section{Results}

Our aim was to determine the variability in RNAi targets to develop an optimal scheme of RNAi for silencing the activity of HIV-1 and to circumvent the problems associated with the high variability of HIV-1 sequences. In this article, the set of sequences obtained for five Russian isolates is treated as a model of an individual patient. Such a set can mimic the variability of RNAi targets for multistrain HIV-1 patients and/or the development of multiple strains over time for a particular patient. As we are primarily interested in assessing the characteristic quantitative variability of RNAi targets, such an approach seems reasonable. To ensure reliable statistics for the rather small set of data, the study of variability was extended to $300 \mathrm{nt}$ fragments that included five $23 \mathrm{nt}$ targets. Positions of the targets are shown in Figure 1 and designated as A1, A2, A3, A4, and A6.

\section{Variations in the vicinity of RNAi targets}

As a convenient reference, we added to our data set the corresponding fragments from the genome sequence for 


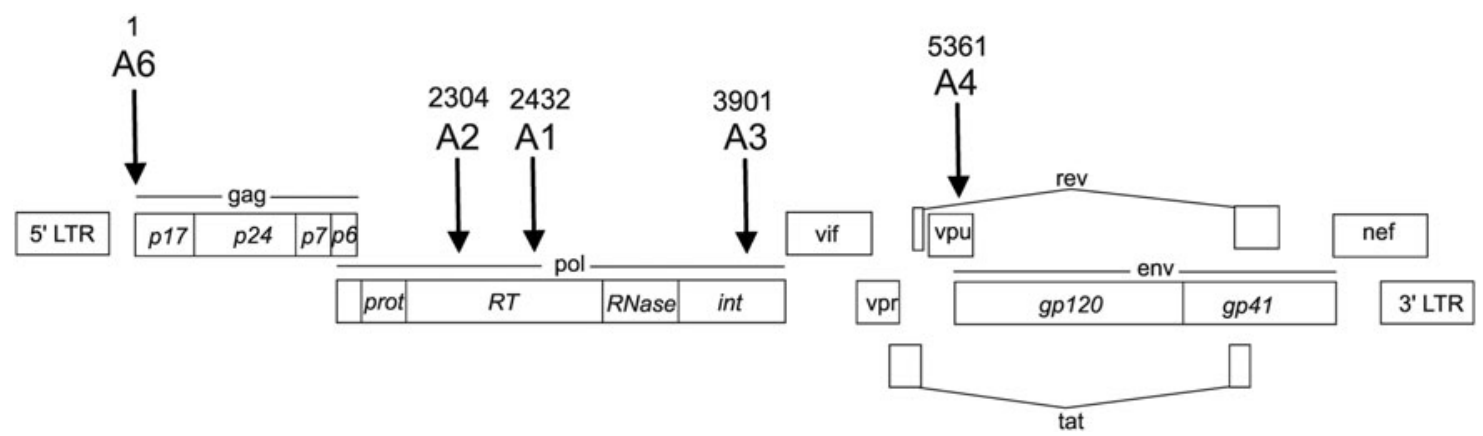

FIG. 1. Schematic presentation of RNAi targets (A1-A5, and A6) within the HIV-1 gene map. The 23-bp targets are located in the sequenced regions of about $300 \mathrm{bp}$ in length in HIV-1 subtype A isolates in Russia. The values shown above the targets indicate the $5^{\prime}$ numbering of a target in the reference sequence (GenBank AF316544). A1-A3 are located inside the pol domain, A4 inside vpu, and A6 inside the gag domain. The corresponding 300 bp sequences comprising the A1-A5 and A6 RNAi targets are shown in Supplementary Fig. S1.

HIV-1 isolate 97CDKP58e from the Republic of the Congo (GenBank Accession No. AF316544; hereafter HIV-1 Congo). The sequence for HIV-1 Congo is commonly considered as a generic reference for HIV-1 subtype A sequences. The general (dis)similarity between $300 \mathrm{nt}$ fragments around RNAi targets for the mixture of five Russian isolates and HIV-1 Congo is shown in Figure 2 and is characterized in terms of maximum-likelihood trees. The trees were obtained by the tools based on the PhyML algorithm ${ }^{7}$ that are built into the UGENE package [8]. The distances on the branches reveal that the 300-nt fragments for HIV-1 Congo are clearly more distant from the counterpart fragments for the five Russian isolates, which are rather close to each other. The details of genetic variations (mutations, stop codons, and microindels) responsible for the divergence between the 300nt fragments around the RNAi targets can be found in the Supplementary Fig. S1 (Supplementary Data are available online at www.liebertpub.com/aid).

The mutations in the sequenced 300-nt fragments around RNAi targets were counted relative to a chosen reference sequence. Preliminarily, all sequences were aligned using the UGENE toolkit. ${ }^{8}$ The frequency of mutations was assessed by

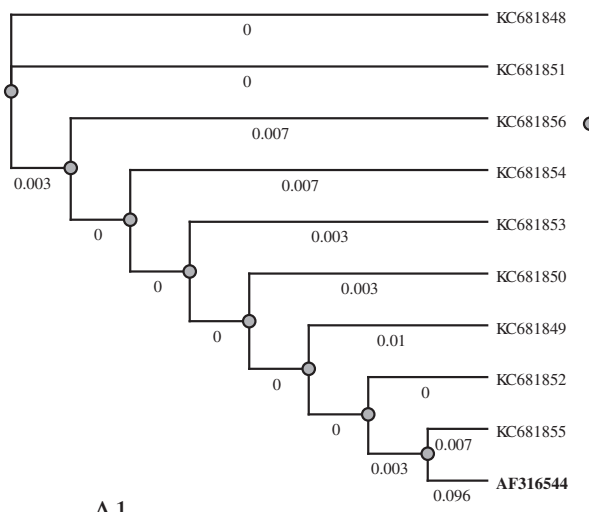

A1

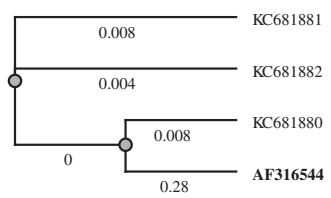

A4

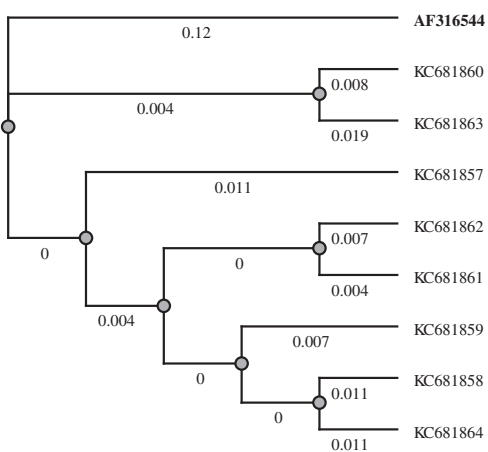

A2

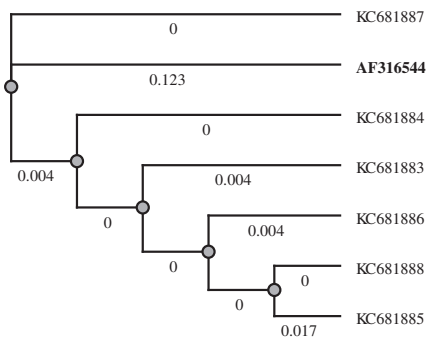

A6

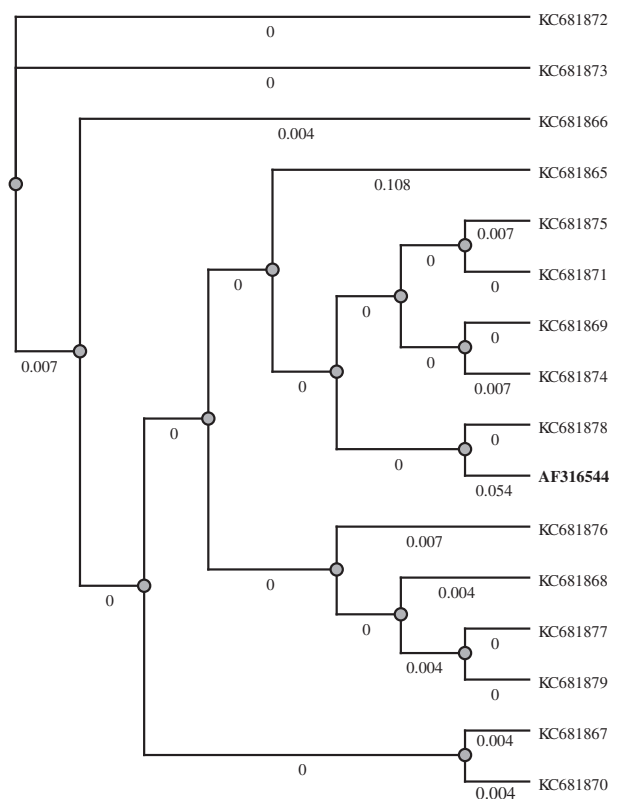

A3

FIG. 2. Maximum-likelihood trees for the 300-nt sequences comprising RNAi targets for the mixture of five Russian HIV-1 subtype A isolates. The corresponding sequences for HIV-1 isolate 97CDKP58e from the Republic of the Congo (AF316544) are boldfaced. A1-A6 refer to RNAi targets. 
two methods. In the first method, the corresponding fragments in the genome sequence for HIV-1 Congo were used as the relevant reference sequences (Supplementary Fig. S1). The frequency of mutations $f_{m}$ is defined in this case as

$$
f_{m}=N_{m} / N_{s} L_{r e f},
$$

where $N_{m}$ is the complete number of mutations detected in the set of 300-nt fragments corresponding to a particular RNAi target, $N_{s}$ is the number of $300 \mathrm{nt}$ fragments, and $L_{r e f}$ is the length of the reference sequence. In the second method, we used jackknife (leave-one-out) resampling ${ }^{9}$ in the set of sequenced $300 \mathrm{nt}$ fragments corresponding to a particular RNAi target. In this procedure, one of the 300-nt fragments is picked as the reference sequence. The mutations are counted in the remaining $N_{s}-1$ fragments relative to the chosen sequence. The frequency of mutations in $N_{s}-1$ fragments is defined similarly as in Equation (1), with the replacement $N_{s} \rightarrow N_{s}-1$. Then, the procedure is reiterated by the complete turnover of $300 \mathrm{nt}$ fragments. Finally, the resulting frequencies are averaged over the chosen reference sequences. The equivalent frequencies using the two methods are compared in Figure 3.

In addition to variations related to point mutations, we also detected a few microindels in the vicinity of RNAi targets. These rare events cannot be used for statistical assessment of corresponding frequencies and provide only rough estimates, that is, $10^{-3}-10^{-4}$ (in the first method) and $10^{-4}-10^{-5}$ (in the second method). The variations related to microindels are two to three orders of magnitude less frequent than that of mutations and are not essential for subsequent estimates.

\section{Mutations in RNAi targets}

The frequencies of mutations determined in the vicinity of RNAi targets can be extrapolated to assess the probability of

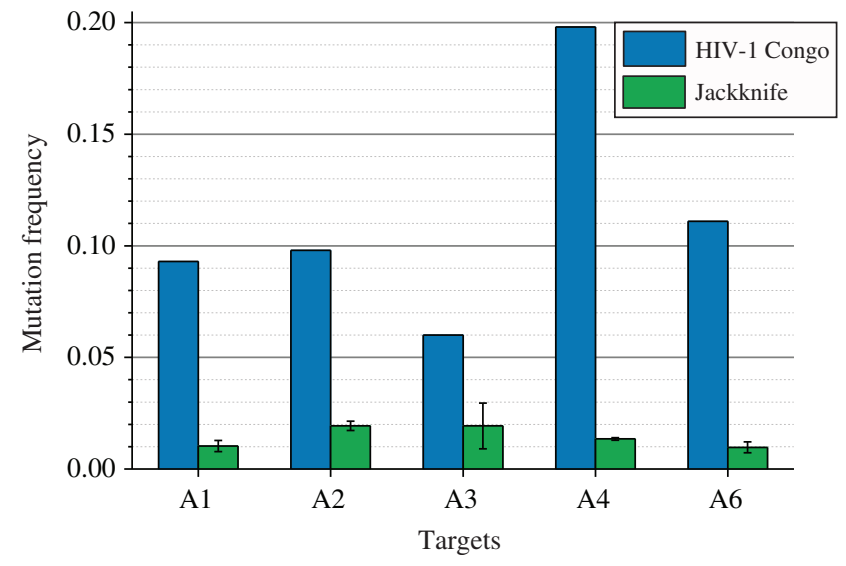

FIG. 3. The mean frequencies of mutations in the 300-nt sequences comprising RNAi targets for the mixture of five Russian HIV-1 subtype A isolates. The frequencies were assessed either relative to the corresponding fragments of HIV-1 isolate 97CDKP58e from the Republic of the Congo (AF316544) or by jackknife resampling of the sets for five Russian isolates, as described in the main text. All sequences were preliminarily aligned using the UGENE toolkit [8]. Color images available online at www.liebertpub.com/aid mutations directly in the targets. Such an extrapolation is much more statistically reliable than direct counting of mutations in the targets for the given set of data. The latter procedure would need data that were several tens of times more extensive.

Assuming Poisson statistics, the probability of detecting $k$ mutations in a target of length $L_{t}$ is given as

$$
P_{k}=\frac{\left(f_{m} L_{t}\right)^{k}}{k !} e^{-f_{m} L_{t}} ; k=0,1,2, \ldots,
$$

where $f_{m}$ corresponds to the frequency of mutations assessed for the set of $300 \mathrm{nt}$ fragments around a particular target. The conservation of the RNAi target corresponds to the absence of mutations, $k=0$. The length of RNAi targets was $L_{t}=23 \mathrm{nt}$. The results of these straightforward computations are summarized in Figure 4.

\section{Multitarget RNAi}

Now we are able to illustrate how the determined probabilities of detecting mutations in RNAi targets (Fig. 4) can be used to choose the optimal treatment for HIV-1 patients. Although the sequence for HIV-1 Congo may be efficiently applied to the assessment of evolutionary divergence between HIV-1 sequences, the corresponding fragments cannot be used as a standard for RNAi targets. RNAi needs the complete correspondence in RNA duplexes, whereas none of the target fragments for HIV-1 Congo remains sufficiently conserved (Figs. 3 and 4).

Consider the reciprocal situation. Let the set of RNAi targets for a particular patient be determined by deep sequencing. Our estimates indicate that these targets will be conserved with a probability in the range of 0.7-0.8 (Fig. 4). The probability $P\left(0 \mid A_{1}, A_{2}, \ldots, A_{n_{t}}\right)$ that at least one target from the $A_{1}, A_{2}, \ldots, A_{n_{t}}$ targets will be completely conserved is expressed through the probabilities $P\left(0 \mid A_{i}\right)$ that targets $A_{i}$ will be conserved as

$$
P\left(0 \mid A_{1}, A_{2}, \ldots, A_{n_{t}}\right)=1-\prod_{i=1}^{n_{t}}\left(1-P\left(0 \mid A_{i}\right)\right) .
$$

For Poisson distribution, the corresponding probabilities are given by $P\left(0 \mid A_{i}\right)=e^{-f_{m, A_{i}} L_{t, A_{i}}}$. For multistrain patients, the targets $A_{i}$ can generally comprise a cluster of close fragments (cf. Supplementary Fig. S1). If any of the targets $A_{1}, A_{2}, \ldots, A_{n_{t}}$ is efficient enough for silencing the activity of HIV-1 through RNAi, multiple targeting may circumvent the effects related to the variability of targets. The dependence of the percentage of patients in which HIV-1 activity can be silenced by RNAi on the number of targets at the different levels of conservation is shown in Figure 5. In particular, for the probability of target conservation of $0.7-0.8$, sets of 3 or 2 targets exceed the threshold of $95 \%$ confidence interval, whereas even for the rather low conservation of 0.4 , sets of six targets may be quite efficient for treatment. Therefore, it may be said that the multiplicity of targets overcomes their variability.

To test this conclusion experimentally, we used the luciferase reporter assay and cloned two targets corresponding to the A1 and A6 regions into the psiCHECK-2 vector (see the corresponding sequences in Supplementary Fig. S1). The 

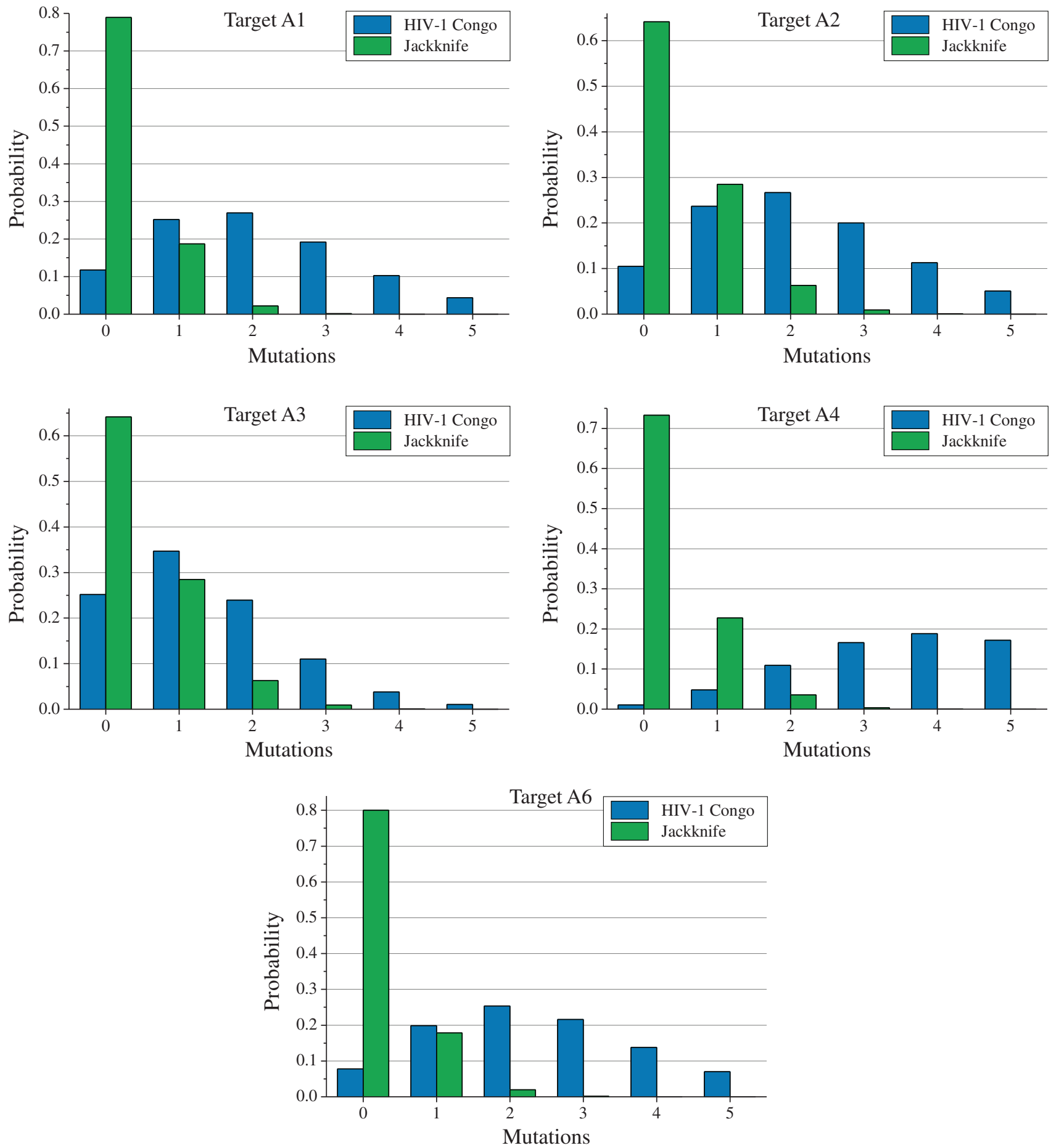

FIG. 4. The probabilities of detecting a fixed number of mutations in the different RNAi targets. The probabilities were calculated by applying Poisson statistics [Eq. (2)] to the targets with a length of $23 \mathrm{nt}$. The mean frequencies of mutations in the RNAi targets were assessed by the number of mutations in the 300-nt sequences comprising RNAi targets for the mixture of five Russian HIV-1 subtype A isolates (Fig. 3). The frequencies were assessed either relative to the corresponding fragments for HIV-1 isolate 97CDKP58e from the Republic of the Congo (AF316544) or by jackknife resampling of the sets for five Russian isolates, as described in the main text. All sequences were preliminarily aligned using the UGENE toolkit [8]. Color images available online at www.liebertpub.com/aid

vector was specifically designed for quantitative measurement of RNAi. XhoI and NotI cloning sites within the 3' UTR of the Renilla luciferase gene were used for cloning of both targets as shown in Figure 6A. Construct 1 possessed an A3 target that perfectly corresponded to the shRNA and an A6 target that contained one mismatch. In contrast, Construct 2 possessed an A3 target with a single mismatch and an A6 target that perfectly matched the corresponding shRNA. Both shRNAs were expressed by the GeneClipU1 plasmid. In cotransfection experiments, we used a mixture of Constructs 1 


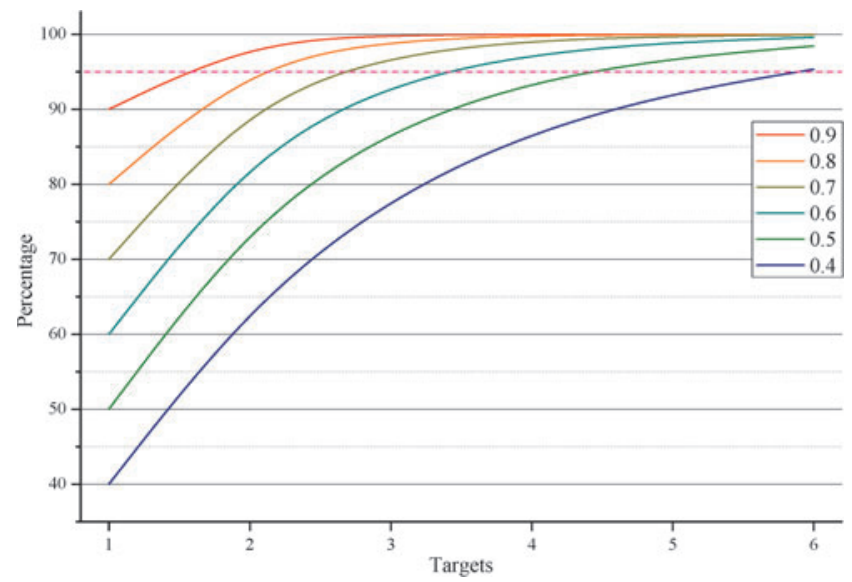

FIG. 5. The dependence of the percentage of patients in which HIV-1 activity can be silenced by RNAi on the number of the RNAi targets with different levels of conservation. The efficiency and conservation of each target are implied to be approximately the same for the particular probability of conservation. The percentage dependencies were calculated by Eq. (3). The horizontal broken line indicates the threshold of $95 \%$ confidence interval recommended by the World Health Organization. Color images available online at www.liebertpub.com/aid

and $2(10 \mathrm{ng}$ each) and the plasmids expressing the shRNAs. The data shown in Figure 6B indicate that, separately, the A3 or A6 siRNA had little effect since a single construct in the mixture could be strongly attacked by the siRNA that perfectly matched the target. In contrast, when both siRNAs were expressed, the efficiency of the RNAi was dramatically in- creased. The data strongly support our conclusion that multiplicity of RNAi targets could overcome their variability.

\section{Discussion}

The HIV-1 retrovirus has acquired a number of molecular mechanisms that allow escape from the protective immunity of the host, as well as current antiretroviral therapy. One of the mechanisms is the high variability of the viral genome, which is mainly due to the error-prone nature of the viral reverse transcriptase. ${ }^{10}$ Ironically, the host-protective mechanism that induces G-to-A substitutions in the viral RNA by deoxycytidine deaminase APOBEC $3 \mathrm{G}$ - which is able to suppress replication of different viruses possessing a singlestranded DNA intermediate by inducing mutagenesis and functional inactivation of the virus-is also a major source of HIV-1 variability. ${ }^{11,12}$ One more source of HIV-1 variability is the recombination-prone nature of the viral reverse transcription machinery that leads to unusually frequent associations between distinct viral genotypes. ${ }^{13,14}$

Our analysis of mutation frequencies in different codon positions in the targets A1-A3 in HIV-1 subtype A isolates in Russia suggests that the most conservative positions are the first and second positions. ${ }^{15}$ The data indicate that there is a positive selection for biologically active viruses in the course of infection.

Whatever mechanism is the cause of frequently occurring mutagenesis in the HIV-1 genome, the monitoring of the sequences of selected RNAi targets should be the first step in the development of RNAi-based gene therapy of HIV/AIDS. Genetic constructs expressing a single or several perfectly matched 21-nt siRNAs in transfected cells lead to efficient attack of the corresponding target in HIV-1. ${ }^{16-19}$ Although a
FIG. 6. Analysis of the efficiency of RNAi initiated by the transfected plasmids expressing shRNAs and the mixture (1:1) of two constructs. (A) The regions of two genetic constructs cloned inside $3^{\prime}$ UTR of Renilla luciferase gene within the psiCHECK-2 vector correspond to A3 and A6 targets (see Supplementary Fig. S1). One perfectly corresponding to siRNA sequence and one possessing a single mismatch were used in each construct. (B) Luciferase assays. shRNAs A3and A6 were expressed from the GeneClipU1 vector. The reporter plasmids (Construct 1 and Construct 2) that were prepared using the psiCHECK-2 vector, containing a pair of luciferase genes, were cotransfected in 1:1 ratio (see the Materials and Methods section). rand., the result obtained with A3 and A6 randomized sequences corresponding to hairpins. $* P<0.05$; $* * P<$ 0.001 . Color images available online at www.liebertpub.com/aid

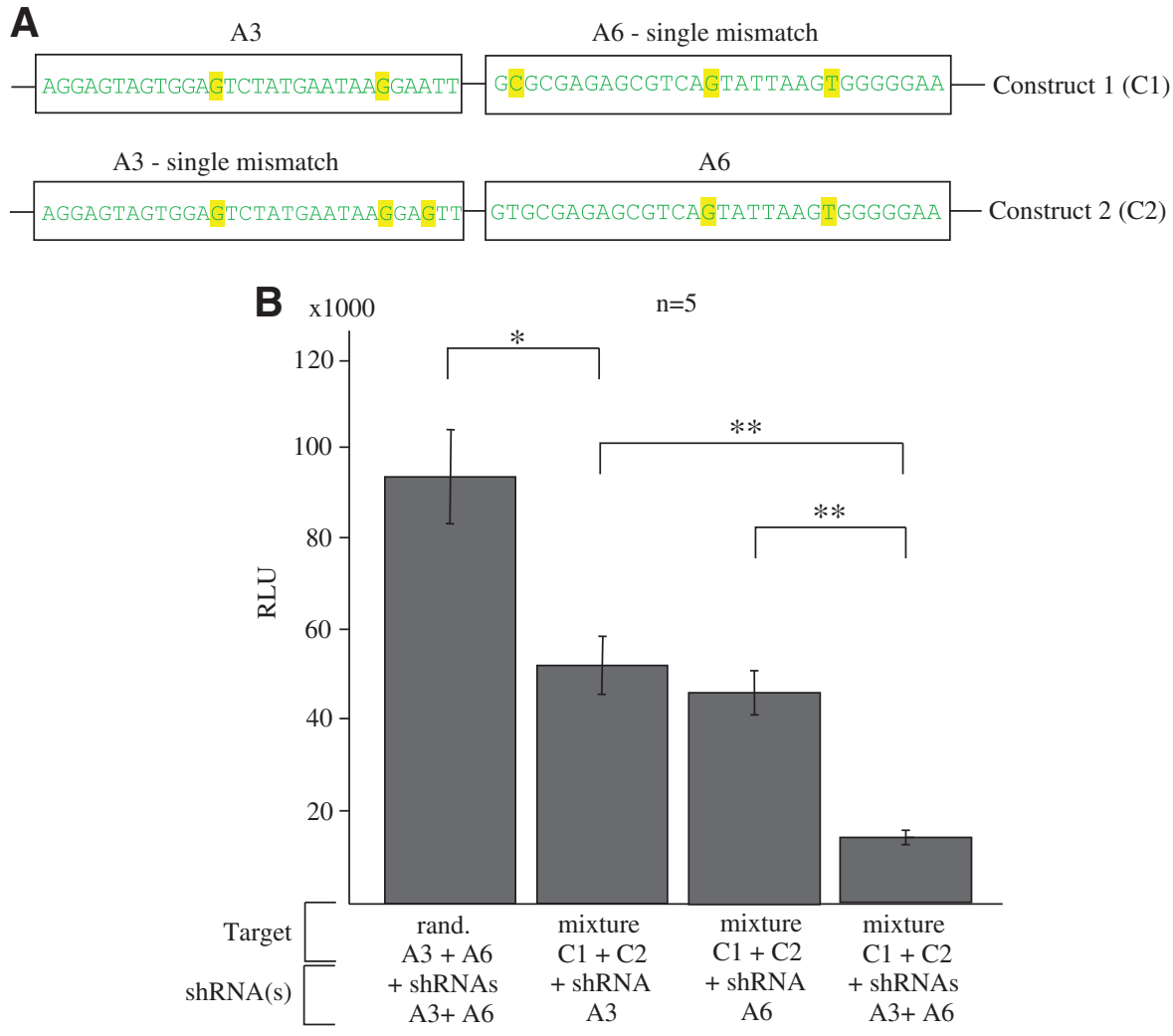


new approach of using the CRISPR gene editing technique was suggested for the removal of HIV from the DNA of human cells, ${ }^{20}$ we believe that RNAi-based technology is closer to practical use. This antiviral technology has a number of advantages over traditional antiviral therapy. It uses an etiological approach as well as highly potent antiviral siRNA molecules as an agent with preventive and therapeutic value. ${ }^{3}$ siRNAs can be designed and synthesized much more easily than traditional antiretroviral drugs. Moreover, a particular siRNA can be used in combination with other siRNAs to reduce the possibility of imperfect correspondence between a single siRNA and its target in the mixture of viruses in a patient.

Multiplying noisy or unreliable elements to improve the functioning of a whole system is a common strategy both in engineering and in living organisms. The greater the variability in the RNAi targets, the larger their number should be. Our estimates indicate that RNAi for silencing of HIV-1 needs at least two targets. Although a fragment can work as an RNAi target only under a set of strict conditions, ${ }^{21}$ the search for two or three targets in the whole genome of HIV-1 seems to not be especially limiting. Duplexes with one or two mismatches cannot participate efficiently in RNAi but are thermodynamically stable and may also partially silence the activity of HIV-1.

The repertoire of RNAi target sequences related to particular subtypes of HIV-1 and/or a particular population should be determined through data stored in available databases $^{22,23}$ or through specific projects. As a medical cure, the RNA duplexes corresponding to the targets for the particular patient can be picked from a pre-synthesized pool of targets that are typical of a particular population. The sequences for targets corresponding to a particular patient should be preliminarily determined before treatment by either deep sequencing or by using custom oligonucleotide microarrays. ${ }^{24-26} \mathrm{We}$ believe that combining deep sequencing and multitarget RNAi may provide an efficient technique to cure HIV/AIDS.

\section{Acknowledgments}

We thank Y.N. Toropchina for technical assistance and Dr. M.P. Gashnikova for the help in some experiments. This work was supported by a grant from the Russian Science Foundation (project no. 15-14-00005).

\section{Author Disclosure Statement}

No competing financial interests exist.

\section{References}

1. Pusch O, Boden D, Silbermann R, Lee F, Tucker L, Ramratnam B: Nucleotide sequence homology requirements of HIV-1-specific short hairpin RNA. Nucleic Acids Res 2003;31:6444-6449.

2. Boden D, Pusch O, Ramratnam B: Overcoming HIV-1 resistance to RNA interference. Front Biosci 2007;12:3104-3116.

3. Barik S: siRNA for influenza therapy. Viruses 2010;2:14481457.

4. Butler IF, Pandrea I, Marx PA, Apetrei C: HIV genetic diversity: Biological and public health consequences. Curr HIV Res 2007;5:23-45.
5. Robinson R: Cellular defense enzyme drives exceptionally high rate of mutation in HIV. PLoS Biol 2015;13: e1002252.

6. Alembekov IR, Kretova OV, Tchurikov NA: Analysis of the genetic constructs expressing antiviral siRNAs in the non-viral test system. Vopr Virusol 2011;56:32-35.

7. Guindon S, Dufayard JF, Lefort V, Anisimova M, Hordijk $\mathrm{W}$, Gascuel O: New algorithms and methods to estimate maximum-likelihood phylogenies: Assessing the performance of PhyML 3.0. Syst Biol 2010;59:307-321.

8. Okonechnikov K, Golosova O, Fursov M, Varlamov A, Vaskin Y, Efremov I, Grehov G, Kandrov D, Rasputin K, Syabro M, Tleukenov T: Unipro UGENE: A unified bioinformatics toolkit. Bioinformatics 2012;28:1166-1167.

9. Weir BS: Genetic Data Analysis II: Methods for Discrete Population Genetic Data. Sinauer Associates, Sunderland, MA, 1996.

10. Kim T, Mudry RA, Jr., Rexrode CA, Pathak VK: Retroviral mutation rates and A-to-G hypermutations during different stages of retroviral replication. J Virol 1996;70:7594-7602.

11. Feng Y, Baig TT, Love RP, Chelico L: Suppression of APOBEC3-mediated restriction of HIV-1 by Vif. Front Microbiol 2014;5:450.

12. Cuevas JM, Geller R, Garijo R, Lopez-Aldeguer J, Sanjuan $\mathrm{R}$ : Extremely high mutation rate of HIV-1 in vivo. PLoS Biol 2015;13:e1002251.

13. Onafuwa-Nuga A, Telesnitsky A: The remarkable frequency of human immunodeficiency virus type 1 genetic recombination. Microbiol Mol Biol Rev 2009;73:451-480.

14. Gashnikova NM, Bogachev VV, Baryshev PB, Totmenin AV, Gashnikova MP, Kazachinskaya AG, Ismailova TN, Stepanova SA, Chernov AS, Mikheev VN: A rapid expansion of HIV-1 CRF63_02A1 among newly diagnosed HIV-infected individuals in the Tomsk Region, Russia. AIDS Res Hum Retroviruses 2015;31:456-460.

15. Kravatsky YV, Chechetkin VR, Fedoseeva DM, Gorbacheva MA, Kretova OV, Tchurikov NA: Mutation frequencies in HIV-1 subtype A genomes in the regions containing the efficient RNAi targets. Mol Biol (Mosk) 2016;50:480-485.

16. Kretova OV, Alembekov IR, Tchurikov NA: Generation of genetic constructs that simultaneously express several shRNAs. Biotechniques 2012;52:389-391.

17. Kretova OV, Tchurikov NA: Genetic construct expressing two biologically active siRNAs targeting mRNAs specifying reverse transcriptase and integrase of HIV-1 subtype A from patients in Russia and one siRNA targeting CCR5 mRNA. Patent of Russian Federation 2015; No. 2552486

18. Kretova OV, Tchurikov NA: Genetic construct expressing two biologically active siRNAs targeting the transcripts of HIV-1 subtype A from patients in Russia and one siRNA targeting CCR5 mRNA. Patent of Russian Federation 2015; No. 2552607.

19. Tchurikov NA, Fedoseeva DM, Gashnikova NM, Sosin DV, Gorbacheva MA, Alembekov IR, Chechetkin VR, Kravatsky YV, Kretova OV: Conserved sequences in the current strains of HIV-1 subtype A in Russia are effectively targeted by artificial RNAi in vitro. Gene 2016;583: 78-83.

20. Kaminski R, Chen Y, Fischer T, Tedaldi E, Napoli A, Zhang Y, Karn J, Wenhui Hu W, Khalili K: Elimination of HIV-1 genomes from human T-lymphoid cells by CRISPR/ Cas9 gene editing. Sci Rep 2016;6:22555. 
21. Reynolds A, Leake D, Boese Q, Scaringe S, Marshall WS, Khvorova A: Rational siRNA design for RNA interference. Nat Biotechnol 2004;22:326-330.

22. Tyagi A, Ahmed F, Thakur N, Sharma A, Raghava GP, Kumar M: HIVsirDB: A database of HIV inhibiting siRNAs. PLoS One 2011;6:e25917.

23. Sharma D, Priyadarshini P, Vrati S: Unraveling the web of viroinformatics: Computational tools and databases in virus research. J Virol 2015;89:1489-1501.

24. Hacia JG, Collins FS: Mutational analysis using oligonucleotide microarrays. J Med Genet 1999;36:730-736.

25. Chechetkin VR, Prokopenko DV, Makarov AA, Zasedatelev AS: Biochips for medical diagnostics. Russ Nanotechnol 2006;1:13-27.
26. Mehla R, Ayyavoo V: Gene array studies in HIV-1 infection. Curr HIV/AIDS Rep 2012;9:34-43.

Address correspondence to: Nickolai A. Tchurikov Engelhardt Institute of Molecular Biology Russian Academy of Sciences Vavilov Street 32 Moscow 119334 Russia

E-mail: tchurikov@eimb.ru 